\title{
Ethnic Violence and Community Participation in Indonesia
}

\author{
${ }^{1}$ Christophe Muller, and ${ }^{2}$ Marc Vothknecht \\ ${ }^{1}$ Aix-MarseilleUniversity (Aix-Marseille School of Economics), EHESS \&CNRS, \\ 14 Avenue Jules Ferry, 13621 Aix en Provence, France. \\ ${ }^{2}$ European Commission
}

\begin{abstract}
We Study The Impact Of Violent Conflict On Local Participation In Governance, Social Service, Infrastructure Development And Risk-Sharing, In Connection With Local Ethnic Diversity. Social Capital Is Measured By Citizen Participation In Four Kinds Of Community Groups.

Combining household panel data from Indonesia with conflict event information, we find an overall decrease in participation in districts affected by group violence in the post-Suharto transition period. However, participation is found to be little affected by violence in communities with a high degree of ethnic polarization, and is even stimulated for local governance and risk-sharing activities.
\end{abstract}

Keywords: Violent Conflict, Ethnic Diversity, Citizen Participation, Local Public Goods. JEL Codes: D74, H42, O11.

\section{Introduction}

Local community groups are often believed to be useful to overcome shortages in the provision of local public goods and services in developing economies. In the absence of formal credit and insurance markets, networks of mutual assistance may spur productive investments and mitigate income shocks. Finally, information dissemination and political decisions often take place within local organizations.

The link between violence in the immediate post-Suharto era and participation in local community groups has not been analysed quantitatively in Indonesia. We fill this gap with hard empirical evidence in this paper.

The next section describes the data and provides background information on community activities in Indonesia. We discuss our estimation strategy in Section 3. In Section 4, we represent our empirical results. Finally, Section 5 concludes.

\section{The Context and the Data}

We study the functioning of these local groups by using data from the Indonesian Family Life Survey (IFLS), a large-scale, longitudinal household and community survey representative of about 83 per cent of the Indonesian population. The second (IFLS2 in 1997) and the third wave (ILFS3 in 2000) that we use allows us to capture information contemporary to the 1997 financial crisis and the outbreak of violence in the aftermath of President Suharto's resignation in May 1998.

Since the conflict data we draw on is not available for those Indonesian provinces with negligible levels of communal violence, our analysis focuses on the main island of Java, the islands of West Nusa Tenggara, and the province of South Sulawesi. The community-level activitiesare grouped into four (mutually non-exclusive) categories: local governance organizations, social services, infrastructure development initiatives and mutual insurance groups. 
For the quantitative analysis of ethnic conflicts, we use the United Nations Support Facility for Indonesian Recovery (UNSFIR)-II Database, which reports incidents of group violence in fourteen Indonesian provinces for 1990-2003.

\section{The Econometric Approach}

The analysis of the determinants of individual participation is conducted separately for each activity category, as well as for security organizations and cooperatives. The propensity of individual $i$ to participate in a certain community activity, $k$,in community $j$ and year $t$ is dependent on the expected net benefit from involvement, $\mathrm{B}^{*}$ :

$$
B_{i j t k}^{*}=X_{i t} \beta+V_{j t} \gamma+R_{j} \delta+T_{t} \varphi+a_{i}+v_{t-1, d} \vartheta+\varepsilon_{i t},
$$

where $X_{i t}$ is a vector of individual and household characteristics, $V_{j t}$ a vector of village characteristics, $R_{j}$ and $T_{t}$ are province and time dummies, $a_{i}$ denotes an unobserved individual effect, $\varepsilon_{i t}$ is an idiosyncratic error term with mean zero, and $\beta, \gamma, \delta, \varphi, \vartheta$ represent parameter vectors. Our main independent variable of interest is the indicator of conflict, $v_{t-1, d}$, which measures lagged violence at district level. While the expectations on net benefits are unknown, we observe the individual participation choice, $P_{i t k}$, which equals 1 (participation) if the expected net benefit is positive, and zero (no participation) otherwise:

$$
P_{i t k}=1 \text { if } B_{i j t k}^{*}>0,0 \text { otherwise. }
$$

A Random Effects (RE) logit model is specified to estimate (1)-(2). Thus, the panel structure of the data allows us to account for unobserved individual heterogeneity that might affect individual engagement.

\section{Empirical Results}

We run separate random-effect logit regressions on individual participation for each constructed activity category. Our base regression results are presented in Table 1. Particularly strong effects are observed for age, gender and the individual's position within the household, which point to societal role models that foster or discourage participation in village life. On the one hand, participation in most activities further requires a minimum level of skills. On the other hand, involvement is generally increasing with educational attainment (except for security groups).

Further, citizen participation seems to be driven by specific individual needs related to occupation, family characteristics or special situations, which can all be addressed through different community activities. Recent migrants, as well as members of ethnic minorities, are less likely to participate, especially in governance and risk-sharing activities, perhaps because of different needs or weaker network access. The economically better off are the most likely to be involved in local decision-making, while being less present when it comes to the improvement of local infrastructure.

Finally, we find relatively few effects of village-level variables, which are partly absorbed by the highly significant province dummies and individual random effects. The proportion of the total variance of errors that can be attributed to unobserved individual heterogeneity through individual random effects is substantial. It ranges from $23 \%$ to $60 \%$ depending on the considered activity, with the exception of infrastructure groups. This suggests that many of the decision determinants originate in unobserved individual characteristics that are stable over time.

In the base specification, we include two dummy variables to control for the impact of prevalent violence on citizen engagement: districts with fewer than 10 reported fatalities form the group of "low intensity conflict" districts, while districts with ten or more fatalities are categorized as "high intensity conflict" areas. Overall, the estimated conflict coefficients reveal substantially lower individual involvement in districts affected by violence. This significant negative effect of conflict on civic engagement is found to increase with conflict intensity across almost all activities. The other tried representations of districts by violence severity did not improve inferences. 
We then assess the role of ethnic polarization for community participation in conflict-affected areas by interacting the conflict variables with a dummy variable for high ethnic polarization. Table 2 presents the results for the polarization and conflict variables. The negative impact of communal violence on citizen participation is partly offset in those conflict-affected communities with a high degree of ethnic polarization. In contrast to the overall decrease in participation in local governance organizations and social services in conflict regions (Table 1), citizen participation is found to be hardly affected in villages characterized by a high degree of ethnic polarization. Hence, the negative effect of conflict on community participation is found significantly stronger in ethnically homogeneous areas.

Based on the fitted regression values, the probabilities of participation are calculated for each individual and activity. In a second step, the estimated average participation probability in conflict-affected regions is compared to a counterfactual of "no violence" case. Table 3reports the estimates, disaggregated by low and high conflict intensity and by the degree of ethnic polarization. In areas with low ethnic polarization (Panel I), average participation propensity is estimated to be substantially lower in the presence of group violence (down to 15 percentage points lower in high intensity conflict areas as compared to the counterfactual "no violence" case). This effect is strongest for social services, security groups, and governance activities, whereas cooperatives seem to be hardly affected by violence.

However, a different picture emerges in areas with a high degree of ethnic polarization (Panel II). In the presence of group violence, average participation probabilities in polarized communities decrease comparably little. In particular, participation in community meetings is barely affected, and the estimates even point to increasing involvement in cooperatives in those districts most affected by violence. Hence, the generally negative impact of communal violence on citizen engagement at the local level is not observed in areas with a high degree of ethnic polarization. Participation in some community groups may even rise in the wake of violent conflict.

TABLE I: Base Random Effect Logit Regression Results

\begin{tabular}{|c|c|c|c|c|c|}
\hline \multirow{2}{*}{$D V: \underline{\text { Participation }}$} & $(1)$ & $(2)$ & $(3)$ & (4) & (5) \\
\hline & Governance & Social Service & Infrastructure & Security & Cooperatives \\
\hline \multicolumn{6}{|l|}{ Individual Characteristics } \\
\hline Age Group: 25-39 Years ${ }^{\mathrm{a}}$ & $\begin{array}{l}0.114^{* * * *} \\
(0.000)\end{array}$ & $\begin{array}{l}0.063^{* * * *} \\
(0.009)\end{array}$ & $\begin{array}{l}0.059^{* *} \\
(0.047)\end{array}$ & $\begin{array}{l}0.180^{* * * *} \\
(0.000)\end{array}$ & $\begin{array}{c}0.065^{* * *} \\
(0.002)^{* * *}\end{array}$ \\
\hline Age Group: 40-65 Years & $\begin{array}{l}0.155^{* * *} \\
(0.000)\end{array}$ & $\begin{array}{l}-0.068^{* *} \\
(0.021)\end{array}$ & $\begin{array}{l}0.095^{*} \\
(0.052)\end{array}$ & $\begin{array}{l}0.150^{* * *} \\
(0.006)\end{array}$ & $\begin{array}{c}0.122 \\
(0.001)\end{array}$ \\
\hline Age Group: >65 Years & $\begin{array}{l}0.119^{* * *} \\
(0.042)\end{array}$ & $\begin{array}{l}-0.107^{* *} \\
(0.034)\end{array}$ & $\begin{array}{c}0.080 \\
(0.209)\end{array}$ & $\begin{array}{l}-0.101 \\
(0.353)\end{array}$ & $\begin{array}{c}0.175 \\
(0.175)\end{array}$ \\
\hline Male & $\begin{array}{c}0.354^{\text {***** }} \\
(0.000)\end{array}$ & & & & $\begin{array}{l}-0.010 \\
(0.138)\end{array}$ \\
\hline No education $^{\mathrm{b}}$ & $\begin{array}{c}-0.145^{* * * *} \\
(0.000)\end{array}$ & $\begin{array}{c}-0.147^{* * *} \\
(0.000)\end{array}$ & $\begin{array}{l}-0.038 \\
(0.361)\end{array}$ & $\begin{array}{l}-0.119^{* *} \\
(0.018)\end{array}$ & $\begin{array}{c}-0.021^{* * *} \\
(0.006)\end{array}$ \\
\hline Junior High School & $\begin{array}{c}0.066^{* * * *} \\
(0.001)\end{array}$ & $\begin{array}{l}0.057^{* * *} \\
(0.021)\end{array}$ & $\begin{array}{l}-0.040 \\
(0.125)\end{array}$ & $\begin{array}{l}-0.068^{* * *} \\
(0.049)\end{array}$ & $\begin{array}{c}0.014 \\
(0.187)\end{array}$ \\
\hline Senior High School & $\begin{array}{l}0.101^{* * * *} \\
(0.000)\end{array}$ & $\begin{array}{l}0.057^{* * *} \\
(0.030)\end{array}$ & $\begin{array}{l}-0.026 \\
(0.333)\end{array}$ & $\begin{array}{l}-0.078^{* *} \\
(0.025)\end{array}$ & $\begin{array}{l}0.038^{* * * *} \\
(0.009)\end{array}$ \\
\hline Higher Education & $\begin{array}{l}0.111^{* * *} \\
(0.003)\end{array}$ & $\begin{array}{c}0.012 \\
(0.806)\end{array}$ & $\begin{array}{l}-0.004 \\
(0.923)\end{array}$ & $\begin{array}{l}-0.039 \\
(0.508)\end{array}$ & $\begin{array}{l}0.063^{\text {** }} \\
(0.049)\end{array}$ \\
\hline Job Category: Private Worker ${ }^{\mathrm{c}}$ & $\begin{array}{c}0.033 \\
(0.302)\end{array}$ & $\begin{array}{c}0.028 \\
(0.504)\end{array}$ & $\begin{array}{l}0.140^{* * * *} \\
(0.001)\end{array}$ & $\begin{array}{c}0.177^{* * *} \\
(0.000)\end{array}$ & $\begin{array}{c}0.034 \\
(0.114)\end{array}$ \\
\hline Job Category: Self-Employed & $\begin{array}{l}0.064^{* * *} \\
(0.038)\end{array}$ & $\begin{array}{l}0.083^{* *} \\
(0.043)\end{array}$ & $\begin{array}{l}0.149^{* * * *} \\
(0.000)\end{array}$ & $\begin{array}{c}0.138^{* * * *} \\
(0.006)\end{array}$ & $\begin{array}{c}0.022 \\
(0.231)\end{array}$ \\
\hline $\begin{array}{l}\text { Job Category: Unpaid Family } \\
\text { Worker }\end{array}$ & $\begin{array}{c}0.009 \\
(0.731)\end{array}$ & $\begin{array}{c}0.011 \\
(0.703)\end{array}$ & $\begin{array}{l}0.125^{* * *} \\
(0.000)\end{array}$ & $\begin{array}{c}0.006 \\
(0.912)\end{array}$ & $\begin{array}{l}-0.002 \\
(0.863)\end{array}$ \\
\hline Job Category: Government & $\begin{array}{l}0.164^{* * *} \\
(0.001)\end{array}$ & $\begin{array}{l}0.198^{* * *} \\
(0.004)\end{array}$ & $\begin{array}{l}0.161^{* * *} \\
(0.000)\end{array}$ & $\begin{array}{l}0.179^{* * *} \\
(0.000)\end{array}$ & $\begin{array}{l}0.189^{* *} \\
(0.030)\end{array}$ \\
\hline
\end{tabular}




\begin{tabular}{|c|c|c|c|c|c|}
\hline Hours worked per week & $\begin{array}{l}-0.001 \\
(0.697)\end{array}$ & $\begin{array}{l}-0.016^{* * *} \\
(0.000)\end{array}$ & $\begin{array}{l}-0.006 \\
(0.182)\end{array}$ & $\begin{array}{c}0.007 \\
(0.254)\end{array}$ & $\begin{array}{c}0.000 \\
(0.967)\end{array}$ \\
\hline Total monthly income (ln) & $\begin{array}{c}0.001 \\
(0.464)\end{array}$ & $\begin{array}{l}-0.004 \\
(0.151)\end{array}$ & $\begin{array}{c}0.002 \\
(0.474)\end{array}$ & $\begin{array}{l}-0.005 \\
(0.152)\end{array}$ & $\begin{array}{l}-0.001 \\
(0.398)\end{array}$ \\
\hline Married & $\begin{array}{l}0.147^{* * * *} \\
(0.000)\end{array}$ & $\begin{array}{l}0.382^{* * *} \\
(0.000)\end{array}$ & $\begin{array}{c}0.047 \\
(0.187)\end{array}$ & $\begin{array}{l}0.100^{* *} \\
(0.018)\end{array}$ & $\begin{array}{c}0.009 \\
(0.313)\end{array}$ \\
\hline Head or Spouse of Head & $\begin{array}{l}0.138^{* * *} \\
(0.000)\end{array}$ & $\begin{array}{c}0.022 \\
(0.409)\end{array}$ & $\begin{array}{c}0.022 \\
(0.643)\end{array}$ & $\begin{array}{l}0.118^{* *} \\
(0.042)\end{array}$ & $\begin{array}{l}0.023^{* *} \\
(0.045)\end{array}$ \\
\hline $\begin{array}{l}\text { Population Share of one's own } \\
\text { Ethnicity in the Village }\end{array}$ & $\begin{array}{l}0.129^{* * *} \\
(0.001)\end{array}$ & $\begin{array}{l}0.100^{* *} \\
(0.028)\end{array}$ & $\begin{array}{c}0.075 \\
(0.109)\end{array}$ & $\begin{array}{l}0.097^{*} \\
(0.097)\end{array}$ & $\begin{array}{c}0.027 \\
(0.135)\end{array}$ \\
\hline $\begin{array}{l}\text { Seriousness of Answers: } \\
\text { not excellent or good }\end{array}$ & $\begin{array}{l}-0.009 \\
(0.542)\end{array}$ & $\begin{array}{l}-0.032^{*} \\
(0.065)\end{array}$ & $\begin{array}{l}-0.051^{* *} \\
(0.025)\end{array}$ & $\begin{array}{c}0.009 \\
(0.738)\end{array}$ & $\begin{array}{c}0.006 \\
(0.418)\end{array}$ \\
\hline \multicolumn{6}{|l|}{ Household Characteristics } \\
\hline Age HH Head: 40-65 Years ${ }^{\mathrm{a}}$ & $\begin{array}{c}0.022 \\
(0.257)\end{array}$ & $\begin{array}{l}-0.112^{* * *} \\
(0.000)\end{array}$ & $\begin{array}{l}-0.061 \\
(0.116)\end{array}$ & $\begin{array}{c}0.009 \\
(0.846)\end{array}$ & $\begin{array}{l}-0.012 \\
(0.273)\end{array}$ \\
\hline Age HH Head: >65 Years & $\begin{array}{c}0.014 \\
(0.643)\end{array}$ & $\begin{array}{l}-0.143^{* * *} \\
(0.000)\end{array}$ & $\begin{array}{l}-0.069 \\
(0.245)\end{array}$ & $\begin{array}{c}0.006 \\
(0.916)\end{array}$ & $\begin{array}{c}-0.021 \\
(0.023)^{* *}\end{array}$ \\
\hline $\begin{array}{l}\text { Household Expenditure - } \\
1^{\text {st }} \text { Quantile }^{\mathrm{d}}\end{array}$ & $\begin{array}{l}-0.051^{* * *} \\
(0.000)\end{array}$ & $\begin{array}{l}-0.010 \\
(0.554)\end{array}$ & $\begin{array}{l}-0.001 \\
(0.979)\end{array}$ & $\begin{array}{l}-0.023 \\
(0.397)\end{array}$ & $\begin{array}{l}-0.005 \\
(0.493)\end{array}$ \\
\hline $\begin{array}{l}\text { Household Expenditure - } \\
4^{\text {th }} \text { Quantile }\end{array}$ & $\begin{array}{l}0.079^{* * *} \\
(0.000)\end{array}$ & $\begin{array}{c}0.001 \\
(0.959)\end{array}$ & $\begin{array}{l}-0.050^{* *} \\
(0.045)\end{array}$ & $\begin{array}{c}0.013 \\
(0.666)\end{array}$ & $\begin{array}{c}0.000 \\
(0.986)\end{array}$ \\
\hline $\begin{array}{l}\text { Relative Wealth: Asset Value Rank } \\
\text { within Village }\end{array}$ & $\begin{array}{l}0.088^{* * *} \\
(0.000)\end{array}$ & $\begin{array}{l}-0.037 \\
(0.200)\end{array}$ & $\begin{array}{c}0.009 \\
(0.783)\end{array}$ & $\begin{array}{c}0.006 \\
(0.891)\end{array}$ & $\begin{array}{c}0.019 \\
(0.105)\end{array}$ \\
\hline Household with Farm Income & $\begin{array}{c}0.013 \\
(0.414)\end{array}$ & $\begin{array}{l}-0.040^{* *} \\
(0.032)\end{array}$ & $\begin{array}{l}0.067^{* * *} \\
(0.003)\end{array}$ & $\begin{array}{l}0.048^{*} \\
(0.091)\end{array}$ & $\begin{array}{c}0.010 \\
(0.221)\end{array}$ \\
\hline $\begin{array}{l}\text { Household with Income from Non- } \\
\text { farm Business }\end{array}$ & $\begin{array}{c}0.005 \\
(0.684)\end{array}$ & $\begin{array}{c}0.000 \\
(0.984)\end{array}$ & $\begin{array}{c}0.024 \\
(0.198)\end{array}$ & $\begin{array}{c}0.011 \\
(0.647)\end{array}$ & $\begin{array}{c}0.007 \\
(0.278)\end{array}$ \\
\hline Female Household Head & $\begin{array}{l}0.054^{* *} \\
(0.016)\end{array}$ & $\begin{array}{l}0.172^{* * * *} \\
(0.000)\end{array}$ & $\begin{array}{l}0.099^{* * * *} \\
(0.000)\end{array}$ & $\begin{array}{c}0.036 \\
(0.340)\end{array}$ & $\begin{array}{c}0.006 \\
(0.614)\end{array}$ \\
\hline HH Adults & $\begin{array}{c}0.001 \\
(0.654)\end{array}$ & $\begin{array}{l}0.012^{* * * *} \\
(0.000)\end{array}$ & $\begin{array}{l}-0.004 \\
(0.267)\end{array}$ & $\begin{array}{l}-0.003 \\
(0.552)\end{array}$ & $\begin{array}{l}-0.001 \\
(0.421)\end{array}$ \\
\hline $\begin{array}{l}\text { Recent Economic Hardship } \\
\text { (Crop, Job or Income Loss) }\end{array}$ & $\begin{array}{l}0.031^{* *} \\
(0.020)\end{array}$ & $\begin{array}{l}0.038^{* *} \\
(0.019)\end{array}$ & $\begin{array}{c}0.030 \\
(0.103)\end{array}$ & $\begin{array}{c}0.024 \\
(0.286)\end{array}$ & $\begin{array}{c}0.006 \\
(0.341)\end{array}$ \\
\hline $\begin{array}{l}\text { Household migrated in the last two } \\
\text { yrs to this community }\end{array}$ & $\begin{array}{l}-0.219^{* * * *} \\
(0.000)\end{array}$ & $\begin{array}{l}-0.023 \\
(0.750)\end{array}$ & $\begin{array}{l}-0.017 \\
(0.840) \\
\end{array}$ & $\begin{array}{l}-0.188 \\
(0.174)\end{array}$ & $\begin{array}{l}-0.025^{* *} \\
(0.018)\end{array}$ \\
\hline \multicolumn{6}{|l|}{ Village Characteristics } \\
\hline Rural & $\begin{array}{c}0.005 \\
(0.791)\end{array}$ & $\begin{array}{l}-0.001 \\
(0.998)\end{array}$ & $\begin{array}{l}0.063^{* *} \\
(0.013)\end{array}$ & $\begin{array}{l}-0.056^{*} \\
(0.057)\end{array}$ & $\begin{array}{l}0.016^{*} \\
(0.088)\end{array}$ \\
\hline Population Size & $\begin{array}{l}-0.003 \\
(0.505)\end{array}$ & $\begin{array}{l}-0.006 \\
(0.275)\end{array}$ & $\begin{array}{c}0.005 \\
(0.451)\end{array}$ & $\begin{array}{c}0.004 \\
(0.586)\end{array}$ & $\begin{array}{c}0.001 \\
(0.521)\end{array}$ \\
\hline Average HH Asset Value & $\begin{array}{l}0.024^{* *} \\
(0.025)\end{array}$ & $\begin{array}{l}-0.004 \\
(0.742)\end{array}$ & $\begin{array}{l}-0.035^{* *} \\
(0.020)\end{array}$ & $\begin{array}{l}-0.019 \\
(0.306)\end{array}$ & $\begin{array}{l}-0.007 \\
(0.189)\end{array}$ \\
\hline $\begin{array}{l}\text { Within-Village Gini Index of Asset } \\
\text { Inequality }\end{array}$ & $\begin{array}{l}-0.131 \\
(0.032)\end{array}$ & $\begin{array}{l}0.123^{*} \\
(0.100)\end{array}$ & $\begin{array}{l}-0.028 \\
(0.757)\end{array}$ & $\begin{array}{l}-0.012 \\
(0.914)\end{array}$ & $\begin{array}{l}-0.054^{*} \\
(0.053)\end{array}$ \\
\hline Index of Ethnic Polarization & $\begin{array}{c}0.134^{* * * *} \\
(0.000)\end{array}$ & $\begin{array}{c}0.160^{* * * *} \\
(0.000)\end{array}$ & $\begin{array}{c}0.077^{*} \\
(0.062)\end{array}$ & $\begin{array}{c}0.019 \\
(0.685)\end{array}$ & $\begin{array}{c}0.056^{* * * *} \\
(0.001)\end{array}$ \\
\hline \multicolumn{6}{|l|}{ Conflict Coefficients } \\
\hline Low Intensity: 1-9 Fatalities & $\begin{array}{c}-0.086^{* * *} \\
(0.000)\end{array}$ & $\begin{array}{c}-0.098^{* * *} \\
(0.000)\end{array}$ & $\begin{array}{c}-0.053^{* *} \\
(0.011)\end{array}$ & $\begin{array}{l}-0.046^{*} \\
(0.064)\end{array}$ & $\begin{array}{l}-0.014^{* *} \\
(0.027)\end{array}$ \\
\hline High Intensity: $\geq 10$ Fatalities & $-0.094^{* * *}$ & $-0.129^{* * *}$ & $-0.093^{* *}$ & $-0.107^{*}$ & 0.013 \\
\hline
\end{tabular}




\begin{tabular}{|c|c|c|c|c|c|}
\hline \multicolumn{6}{|l|}{ Province and Time Dummies } \\
\hline Jakarta $^{\mathrm{e}}$ & $\begin{array}{c}-0.144^{* * * *} \\
(0.000)\end{array}$ & $\begin{array}{c}-0.202^{* * *} \\
(0.000)\end{array}$ & $\begin{array}{c}-0.173^{* * *} \\
(0.000)\end{array}$ & $\begin{array}{c}-0.181^{* * *} \\
(0.000)\end{array}$ & $\begin{array}{c}-0.037^{* * *} \\
(0.000)\end{array}$ \\
\hline West Java & $\begin{array}{c}-0.148^{* * * *} \\
(0.000)\end{array}$ & $\begin{array}{c}-0.134^{* * *} \\
(0.000)\end{array}$ & $\begin{array}{l}-0.020 \\
(0.462)\end{array}$ & $\begin{array}{l}-0.052 \\
(0.171)\end{array}$ & $\begin{array}{l}-0.016^{* *} \\
(0.037)\end{array}$ \\
\hline East Java & $\begin{array}{c}-0.053^{* * * *} \\
(0.000)\end{array}$ & $\begin{array}{c}-0.149^{* * *} \\
(0.000)\end{array}$ & $\begin{array}{c}-0.123^{* * *} \\
(0.000)\end{array}$ & $\begin{array}{l}-0.073^{*} \\
(0.087)\end{array}$ & $\begin{array}{l}-0.005^{* *} \\
(0.037)\end{array}$ \\
\hline Nusa Tenggara Barat & $\begin{array}{c}-0.078^{* * * *} \\
(0.000)\end{array}$ & $\begin{array}{l}0.121^{* * * *} \\
(0.000)\end{array}$ & $\begin{array}{c}0.057^{* * *} \\
(0.000)\end{array}$ & $\begin{array}{l}0.023^{*} \\
(0.087)\end{array}$ & $\begin{array}{l}-0.005 \\
(0.626)\end{array}$ \\
\hline South Sulawesi & $\begin{array}{c}-0.158^{* * * *} \\
(0.000)\end{array}$ & $\begin{array}{c}-0.206^{* * * *} \\
(0.000)\end{array}$ & $\begin{array}{c}-0.268^{* * *} \\
(0.000)\end{array}$ & $\begin{array}{l}-0.046 \\
(0.369)\end{array}$ & $\begin{array}{l}-0.016 \\
(0.130)\end{array}$ \\
\hline Year 2000 & $\begin{array}{c}-0.205^{* * *} \\
(0.000)\end{array}$ & $\begin{array}{c}-0.202^{* * * *} \\
(0.000)\end{array}$ & $\begin{array}{c}-0.173^{* * *} \\
(0.000)\end{array}$ & $\begin{array}{c}-0.181^{* * * *} \\
(0.000)\end{array}$ & $\begin{array}{c}-0.053^{* * * *} \\
(0.000)\end{array}$ \\
\hline Observations & 12100 & 8628 & 3414 & 2851 & 3195 \\
\hline Individuals & 8601 & 5481 & 2760 & 2381 & 2754 \\
\hline Average Obs. per Individual & 1.407 & 1.574 & 1.237 & 1.197 & 1.160 \\
\hline Rho & 0.405 & 0.304 & 0.078 & 0.232 & 0.604 \\
\hline
\end{tabular}

RE Logit Regression. Reported: marginal effects at mean values. Conditional on activity existence at village level. Longitudinal personal weights used. $\mathrm{P}$ values in parentheses. * significant at $10 \%$; ** significant at $5 \%$; *** significant at $1 \%$.

${ }^{\mathrm{a}}$ Reference category: Age Group 15-24 Years, ${ }^{\mathrm{b}}$ Reference category: Primary education;

${ }^{\mathrm{c}}$ Reference category: Individuals not working, ${ }^{\mathrm{d}}$ Reference category: 2 nd and 3rd Quantile.

${ }^{\mathrm{e}}$ Reference category: Central Java.

TABLE II: Ethnicity and the Effect of Ethnic Polarization in Conflict Areas

\begin{tabular}{|c|c|c|c|c|c|}
\hline \multirow[b]{2}{*}{$D V: \underline{\text { Participation }}$} & (1) & (2) & (3) & (4) & (5) \\
\hline & Governance & $\begin{array}{l}\text { Social } \\
\text { Service }\end{array}$ & Infrastructure & Security & Cooperatives \\
\hline \multicolumn{6}{|l|}{ Village Characteristics } \\
\hline $\begin{array}{l}\text { Index of Ethnic } \\
\text { Polarization }\end{array}$ & $\begin{array}{l}0.40 * * \\
(0.022)\end{array}$ & $\begin{array}{l}0.49 * * * \\
(0.007)\end{array}$ & $\begin{array}{c}0.23 \\
(0.362)\end{array}$ & $\begin{array}{c}0.25 \\
(0.396)\end{array}$ & $\begin{array}{l}1.18 * * \\
(0.013)\end{array}$ \\
\hline \multicolumn{6}{|l|}{ Conflict Variables } \\
\hline $\begin{array}{l}\text { Low Intensity: 1-9 } \\
\text { Fatalities }\end{array}$ & $\begin{array}{c}-0.51 * * * \\
(0.000)\end{array}$ & $\begin{array}{c}-0.47 * * * \\
(0.000)\end{array}$ & $\begin{array}{c}-0.35 * * * \\
(0.004)\end{array}$ & $\begin{array}{c}-0.13 \\
(0.382)\end{array}$ & $\begin{array}{l}-0.62 * * \\
(0.012)\end{array}$ \\
\hline $\begin{array}{l}\text { High Intensity: } \geq 10 \\
\text { Fatalities }\end{array}$ & $\begin{array}{c}-0.88 * * * \\
(0.000)\end{array}$ & $\begin{array}{c}-0.93 * * * \\
(0.000)\end{array}$ & $\begin{array}{c}-0.40 \\
(0.140)\end{array}$ & $\begin{array}{l}-0.78 * \\
(0.053)\end{array}$ & $\begin{array}{c}-0.85 \\
(0.112)\end{array}$ \\
\hline $\begin{array}{l}\text { IA: Low Intensity } x \\
\text { High Polarization }\end{array}$ & $\begin{array}{l}0.33 * * \\
(0.020)\end{array}$ & $\begin{array}{c}0.22 \\
(0.144)\end{array}$ & $\begin{array}{c}0.28 \\
(0.193)\end{array}$ & $\begin{array}{c}-0.29 \\
(0.240)\end{array}$ & $\begin{array}{c}0.53 \\
(0.205)\end{array}$ \\
\hline $\begin{array}{l}\text { IA: High Intensity } x \\
\text { High Polarization }\end{array}$ & $\begin{array}{l}0.69 * * * \\
(0.005)\end{array}$ & $\begin{array}{l}0.65 * * \\
(0.011)\end{array}$ & $\begin{array}{c}0.01 \\
(0.969)\end{array}$ & $\begin{array}{c}0.31 \\
(0.497)\end{array}$ & $\begin{array}{l}1.96 * * * \\
(0.002)\end{array}$ \\
\hline
\end{tabular}

RE Logit Regression. Reported: coefficient estimates. Apart from the conflict*high polarization interaction variables, same control variables.

TABLE III: Mean Participation Probabilities. Standard Errors in Parentheses.

I. LOW ETHNIC POLARIZATION

\begin{tabular}{l|cc|c|c|c|c}
\hline \multirow{3}{*}{ Activity } & \multicolumn{2}{|c|}{ Low Conflict Intensity Districts } & \multicolumn{3}{c}{ High Conflict Intensity Districts } \\
\cline { 2 - 7 } & $\begin{array}{c}\text { "No Violence" } \\
\text { Counterfactual }\end{array}$ & $\begin{array}{c}\text { Mean } \\
\text { Participation } \\
\text { Probability }\end{array}$ & $\begin{array}{c}\text { Relative Difference: } \\
\text { Violence to Peace } \\
(\%)\end{array}$ & $\begin{array}{c}\text { "No } \\
\text { Violence" } \\
\text { Counterfactual }\end{array}$ & $\begin{array}{c}\text { Mean } \\
\text { Participation } \\
\text { Probability }\end{array}$ & $\begin{array}{c}\text { Relative Difference: } \\
\text { Violence to Peace } \\
(\%)\end{array}$ \\
\hline Local Governance & 34.0 & 26.5 & -7.6 & 29.8 & 18.3 & -11.5 \\
Social Services & $(0.46)$ & $(0.41)$ & $(0.06)$ & $(1.30)$ & $(1.01)$ & $(0.35)$ \\
\hline Infrastructure Development & 44.4 & 35.6 & -8.8 & 38.6 & 23.2 & -15.4 \\
& $(0.47)$ & $(0.44)$ & $(0.05)$ & $(1.48)$ & $(1.14)$ & $(0.41)$ \\
\hline Neighborhood Security Group & 75.6 & 69.6 & -6.1 & 71.0 & 63.4 & -7.7 \\
& $(0.52)$ & $(0.58)$ & $(0.07)$ & $(1.72)$ & $(1.86)$ & $(0.19)$ \\
\end{tabular}




\begin{tabular}{l|cc|c|cc|c} 
& $(0.97)$ & $(0.98)$ & $(0.02)$ & $(3.35)$ & $(3.27)$ & $(0.50)$ \\
\hline Cooperatives & 9.1 & 5.8 & -3.4 & 4.7 & 2.2 & -2.5 \\
& $(0.48)$ & $(0.36)$ & $(0.13)$ & $(0.56)$ & $(0.29)$ & $(0.27)$ \\
\hline
\end{tabular}

II. High ETHNIC POLARIZATION

\begin{tabular}{l|cc|c|cc|c}
\hline \multirow{2}{*}{ Activity } & \multicolumn{2}{|c|}{ Low Conflict Intensity Districts } & \multicolumn{3}{c}{ High Conflict Intensity Districts } \\
\cline { 2 - 7 } & $\begin{array}{c}\text { "No Violence" } \\
\text { Counterfactual }\end{array}$ & $\begin{array}{c}\text { Mean } \\
\text { Participation } \\
\text { Probability }\end{array}$ & $\begin{array}{c}\text { Relative } \\
\text { Difference: } \\
\text { Violence to } \\
\text { Peace (\%) }\end{array}$ & $\begin{array}{c}\text { "No } \\
\text { Violence" }\end{array}$ & $\begin{array}{c}\text { Mean } \\
\text { Counterfactual } \\
\text { Probability }\end{array}$ & $\begin{array}{c}\text { Relative } \\
\text { Difference: } \\
\text { Violence to } \\
\text { Peace (\%) }\end{array}$ \\
\hline \multirow{2}{*}{ Local Governance } & 42.2 & 39.2 & -3.0 & 24.9 & 22.4 & -2.5 \\
Social Services & $(0.78)$ & $(0.77)$ & $(0.03)$ & $(0.69)$ & $(0.65)$ & $(0.04)$ \\
\hline Infrastructure Development & 52.9 & 48.1 & -4.8 & 31.3 & 26.5 & -4.8 \\
& $(0.82)$ & $(0.81)$ & $(0.05)$ & $(0.77)$ & $(0.70)$ & $(0.08)$ \\
\hline \multirow{2}{*}{ Neighborhood Security Group } & 74.0 & 73.0 & -1.0 & 48.7 & 40.3 & -8.4 \\
& $(0.89)$ & $(0.91)$ & $(0.02)$ & $(0.87)$ & $(0.84)$ & $(0.08)$ \\
\hline \multirow{2}{*}{ Cooperatives } & 76.7 & 70.2 & -6.5 & 64.0 & 54.8 & -9.3 \\
& $(1.02)$ & $(1.14)$ & $(0.15)$ & $(1.63)$ & $(1.69)$ & $(0.16)$ \\
\hline
\end{tabular}

TABLE IV: Instrumental Variables: Estimates for the Ethnicity and Conflict Variables

\begin{tabular}{|c|c|c|c|c|c|}
\hline \multirow{2}{*}{$D V: \underline{\text { Participation }}$} & (1) & $(2)$ & $(3)$ & $(4)$ & $(5)$ \\
\hline & Governance & Social Service & Infrastructure & Security & Cooperatives \\
\hline \multicolumn{6}{|c|}{ Conflict and Conflict*Ethnic Polarization-Interaction Variables } \\
\hline \multirow{2}{*}{ Low Intensity: 1-9 Fatalities } & $-0.16^{* * *}$ & $-0.17 * * *$ & -0.11 & -0.10 & -0.07 \\
\hline & $(0.000)$ & $(0.002)$ & $(0.281)$ & $(0.308)$ & $(0.160)$ \\
\hline \multirow{2}{*}{ High Intensity: $\geq 10$ Fatalities } & $-0.45 * * *$ & $-0.46 * * *$ & -0.11 & -0.09 & $-0.20 * *$ \\
\hline & $(0.000)$ & $(0.004)$ & $(0.553)$ & $(0.648)$ & $(0.032)$ \\
\hline \multirow{2}{*}{$\begin{array}{l}\text { IA: Low Intensity x } \\
\text { High Polarization }\end{array}$} & $0.59 * * *$ & $0.65 * * *$ & 0.36 & 0.22 & 0.14 \\
\hline & $(0.000)$ & $(0.000)$ & $(0.218)$ & $(0.351)$ & $(0.189)$ \\
\hline \multirow{2}{*}{$\begin{array}{l}\text { IA: High Intensity x } \\
\text { High Polarization }\end{array}$} & $0.69 * * *$ & $0.69 * * *$ & 0.11 & 0.06 & $0.33 * * *$ \\
\hline & $(0.000)$ & $(0.001)$ & $(0.658)$ & $(0.827)$ & $(0.006)$ \\
\hline
\end{tabular}

Coefficient estimates of RE GLS Regression. The same control variables are included. Instruments included: (1): Share of neighboring districts with 1-9 conflict-related fatalities, (2): share of neighboring districts with 10 or more conflict-related fatalities; (3): IA (1)*high polarization; (4): IA (2)*high polarization; (5)-(8): squared terms of (1)-(4).P-values in parentheses. * significant at 10\%; ** significant at $5 \%$; *** significant at $1 \%$.

We are grateful for comments from participants at many seminars and conferences, in particular from M. Ravallion, M. Rosenzweig, J. Behrman and S. Kalyvas. This research was funded by a MICROCON grant from the European Community's Seventh Framework Programme. The first author is also grateful for support from the A*MIDEX project (No. ANR-11-IDEX-0001-02) funded by the "Investissements d'Avenir" French Government program, managed by the French National Research Agency (ANR). 